\title{
Energy-efficient Trajectory Design for UAV-enabled Wireless Communications with Latency Constraints
}

\author{
Dinh-Hieu Tran*, Thang X. Vu*Member, IEEE, Symeon Chatzinotas*Senior Member, IEEE, \\ and Björn Ottersten*Fellow, IEEE \\ Interdisciplinary Centre for Security, Reliability and Trust (SnT), the University of Luxembourg, Luxembourg. \\ Email: \{hieu.tran-dinh, thang.vu, symeon.chatzinotas, bjorn.ottersten\}@uni.lu
}

\begin{abstract}
This paper studies a new energy-efficient unmanned aerial vehicle (UAV)-enabled wireless communications, where the UAV acts as a flying base station (BS) to serve the ground users (GUs) within some predetermined latency limitations, e.g., requested timeout (RT). Our goal is to design the UAV trajectory to minimize the total energy consumption while satisfying the RT requirement from every GU, which is accomplished via two consecutive subproblems: traveling time minimization and energy minimization problems. Firstly, we propose two exhaustive search and heuristic algorithms based on the traveling salesman problem with time window (TSPTW) in order to minimize the UAV's traveling time without violating the GUs' RT requirements. While the exhaustive algorithm achieves the best performance at a high computation cost, the heuristic algorithm achieves a trade-off between the performance and complexity. Secondly, we minimize the total energy consumption, for a given trajectory, via a joint optimization of the UAV's velocity along subsequent hops. Finally, numerical results are presented to demonstrate the effectiveness of our proposed algorithms. In particular, it is shown that the proposed solutions outperform the reference in terms of both energy consumption and outage performance.
\end{abstract}

Index Terms-UAV, trajectory design, energy minimization, TSPTW.

\section{INTRODUCTION}

With the proliferation of mobile devices and data-hungry applications, the next generation wireless networks are expected to support not only the unprecedented traffic increase and stringent latency but also ubiquitous coverage requirements. Although heterogeneous networks (HetNets) [1] and cloud radio access networks (C-RANs) [2], [3] have shown their capability in supporting massive network traffics, their deployments are usually focused on dense areas. In less-dense areas, e.g., urban, and places where the network traffic highly fluctuates, the employment of C-RANs is economically inefficient. In such cases, the current terrestrial network architecture might suffer network congestion or is insufficient to support the ubiquitous coverage.

Recently, unmanned aerial vehicles (UAVs) have attracted much attention as a promising solution for improving the performance of terrestrial wireless communication networks thanks to their mobility, agility, and flexible deployment [4]. By employing as a flying base station, UAV can be deployed along with ground base stations (GBSs) to provide pervasive coverage and timely applications to ground users (GUs). Consequently, the deployment of UAV in wireless communications has found applications in various domains, such as disaster rescue mission [5], surveillance [6], and smart farming [7]. Besides many advantages, UAV-enabled communications are not without limitation. The inherent natural of UAV has imposed technical restrictions in size, weight, and power capability (SWAP), which consequently affect the UAV's endurance and performance [8]. One of the major challenges in UAV deployment is to efficiently design the trajectory in order to maximize the UAV's service lifetime.

Certain efforts have recently been devoted to efficient UAV trajectory design [8]-[11]. In [9], the authors consider the joint problem of the sensor nodes' wake-up schedule and the trajectory to minimize the maximum energy consumption while guaranteeing the reliability of the data collected from the sensors. Yang et al in [10] analyze the trade-off between uplink transmission energy at GUs and the propulsion energy consumption of UAV. By considering two practical UAV trajectories, namely, circular flight and straight flight, the authors investigate the different Pareto efficiency between the optimal GU transmit power and UAV trajectory design. In [8], the authors design the trajectory of UAV to minimize the mission completion time in UAV-enabled multi-casting systems based on the traveling salesman problem (TSP). In [11], the authors propose a new path discretization method to obtain the optimum trajectory that minimizes the total energy consumption. Note that these works consider the UAV communications without the GUs' transmission constraints, e.g., latency requirement or user quality of experience.

In this paper, we consider UAV-enabled communications systems in practical scenarios in which the GUs' transmissions are subject to some latency or request timeout (RT) constraints. The considered system is motivated from realistic applications, e.g., content delivery networks [12] or age of information, in which when a GU requests a content data, it needs to be served in a certain RT. Our goal is to design energy-efficient UAV trajectory while guaranteeing the predefined RT constraints of all GUs. The considered system is clearly different from [8] which does not consider GUs RT constraint. Our contributions are as follows:

- Firstly, we design the UAV trajectory in order to minimize the UAV traveling time and satisfy the RT constraints for all GUs. In order to deal with the nature NP-hard of the formulated problem, we propose two algorithms, namely, exhaustive search and heuristic algorithms based on the TSPTW method. While the exhaustive search algorithm 
provides the global optimality, its exponential computation complexity might limit its applicability in practical applications. In such cases, the heuristic algorithm with lower complexity can balance the performancecomplexity trade-off.

- Secondly, we minimize the total UAV's energy consumption for a given trajectory via a joint optimization of the $\mathrm{UAV}$ velocities in all hops. The formulated problem is proved to be convex and hence be efficiently solved by the standard method.

- Finally, the effectiveness of the proposed algorithms are demonstrated via numerical results, which show significant improvements in both energy consumption and outage probability compared with the conventional design [11].

Notations: For a set $\mathcal{K},|\mathcal{K}|$ denotes its cardinality. For a vector $v,\|v\|$ denotes its Euclidean norm . For a function $f(x), \nabla f(x)$ represents the gradient of $f(x) .1_{m, n}$ is the $m \times n$ matrix of ones where every element equals to one.

\section{SYSTEM MODEL}

We consider a UAV-enabled communication system in which a UAV helps to serve a set $\mathcal{K}=\{1, \ldots, K\}$ consisting of $K$ GUs. Due to limited access, the users can only receive data from the UAV [8], [11]. The location of GU $k$ is denoted as $q_{k} \in \mathbb{R}^{2 \times 1}, k \in \mathcal{K}$. Let $\left(u_{1}, u_{2}, \ldots, u_{K}\right)$ be a permutation of $(1,2, \ldots, K)$, and let $\mathbf{u} \triangleq\left[u_{1}, u_{2}, \ldots, u_{K}\right]$ specify a trajectory of the UAV to serve all users following the path $0 \rightarrow u_{1} \rightarrow u_{2} \rightarrow \ldots \rightarrow u_{K} \rightarrow 0$, where 0 denotes the UAV station (or depot). The UAV is assumed to employ the hovering-communication method [8] to serve the GUs. In this model, in order to serve GU $k$, the UAV has to move to GU $k$ 's location and keeps hovering during the transmission period ${ }^{1}$. Fig. 1a depicts a two-dimensional Cartesian coordinate system and different trajectory designs. Furthermore, denote $\eta_{k}$ as the $\mathrm{RT}$ of $\mathrm{GU} k, \forall k \in \mathcal{K}$.

\section{A. Transmission model}

The UAV's trajectory is split into $K+1$ line segments (or hops) which are represented by all connections between $K+$ 2 way-points on the selected route (see Fig. 1 for details). We assume the UAV fly at a constant altitude of $H$ (meters). Therefore, the distance of the $k$-th hop is given as

$$
l_{j \rightarrow k}=\left\|q_{j}-q_{k}\right\|, 0 \leq j, k \leq K,
$$

where the index 0 represents the UAV station and $\|q\|$ represents the Euclidean distance of vector $q$. We assume that the UAV flies at a constant velocity during one hop but can change from hop to hop. Denote $v_{k}$ is the UAV velocity at the $k$-th hop. In addition, let $\tau_{k}$ as the transmission time needed for UAV to send the requested data to GU $k$ reliably. Then the time for the $\mathrm{UAV}$ to reach the $k \mathrm{GU}$ is calculated as

$$
T_{k}=\sum_{i=1}^{k}\left(\frac{l_{i}}{v_{i}}+\tau_{i}\right), 1 \leq k \leq K,
$$

\footnotetext{
${ }^{1}$ Consider other transmission models, e.g., flying-communicate, is left for future work.
}

where $t_{k}=l_{k} / v_{k}$ represents the travel time in the $k$-th hop and $\tau_{0} \triangleq 0$ for convenience.

The transmission time to serve GU $k$ is computed as $\tau_{k}=$ $Q_{k} / R_{k}$, where $Q_{k}$ denotes the length in bits of the requested content and $R_{k}$ denotes the transmission rate from the UAV to GU $k$. Under the hovering-communicate method, the UAVGU link is dominated by LoS link [13]. Consequently, the communication rate is computed as follows:

$$
R_{k}=B \log _{2}\left(1+\frac{P_{\text {com }}}{H^{\alpha} \sigma^{2}}\right),
$$

where $B$ is the channel bandwidth, $P_{\text {com }}$ is the transmit power for the UAV, $H^{\alpha}$ represents the pathloss, $\alpha$ is the pathloss exponent, and $\sigma^{2}$ is the noise power. In order to satisfy the quality of service (QoS) constraint, it must hold $R_{k} \geq r_{k}, \forall k$, where $r_{k}$ is the QoS constraint of GU $k$.

\section{B. Energy consumption model}

The energy consumption of the UAV consists of two types: propulsion energy consumption and communication energy consumption. The former measures the energy consumed to fly or hover the UAV. The latter is used to transmit data to the GUs.

The power consumption of a rotary-wing UAV flying at velocity $v$ is given as [11, Eq. (6)]

$$
\begin{aligned}
P_{f l y}(v)= & \underbrace{P_{0}\left(1+\alpha_{1} v^{2}\right)}_{\text {bladeprofile }} \\
& +\underbrace{P_{1} \sqrt{\sqrt{1+\alpha_{2}^{2} v^{4}}-\alpha_{2} v^{2}}}_{\text {induced }}+\underbrace{\alpha_{3} v^{3}}_{\text {parasite }} .
\end{aligned}
$$

where $P_{0}=\frac{\delta}{8} \rho s A \Omega^{3} R^{3}, P_{1}=(1+I) \frac{W^{3 / 2}}{\sqrt{2 \rho A}}, \alpha_{1}=\frac{3}{\Omega^{2} R^{2}}$, $\alpha_{2}=\frac{1}{2 V_{R}^{2}}$, and $\alpha_{3}=0.5 d_{0} \rho s A$. Blade profile power, parasite power, and induced power are needed to overcome the profile drag of the blades, the fuselage drag, the induced drag of the blades, respectively. Other parameters are explained as in Table I.

TABLE I: Notations for rotary-wing UAV

\begin{tabular}{|l|l|l|}
\hline Notations & Meanings & Values \\
\hline$\delta$ & The profile drag coefficient & 0.012 \\
\hline$\rho$ & Air density in $\mathrm{kg} / \mathrm{m}^{3}$ & 1.225 \\
\hline$R$ & Rotor radius in meter & 0.5 \\
\hline$A$ & Rotor disc area in $\mathrm{m}^{2}, A \triangleq \pi * R^{2}$. & 0.785 \\
\hline$s$ & Rotor solidity & 0.0499 \\
\hline$\Omega$ & Blade angular velocity in radians/second & 400 \\
\hline$W$ & UAV weight in Newton & 100 \\
\hline$I$ & Thrust-to-weight ratio & 1 \\
\hline$V_{R}$ & The mean rotor induced velocity in hover & 7.2 \\
\hline$d_{0}$ & Fuselage drag ratio & 0.3 \\
\hline
\end{tabular}

The total energy consumption that the UAV spends on hop $k$ is given as

$$
E_{k}\left(v_{k}\right)=E_{f l y, k}+E_{h o v, k}+E_{c o m, k},
$$

where $E_{f l y, k}=P_{f l y}\left(v_{k}\right) \times t_{k}, E_{h o v, k}=P_{f l y}\left(v_{h o v}\right) \times t_{k}$, and $E_{c o m, k}=P_{c o m} \times \tau_{k}$ are the energy consumption due to flying, 


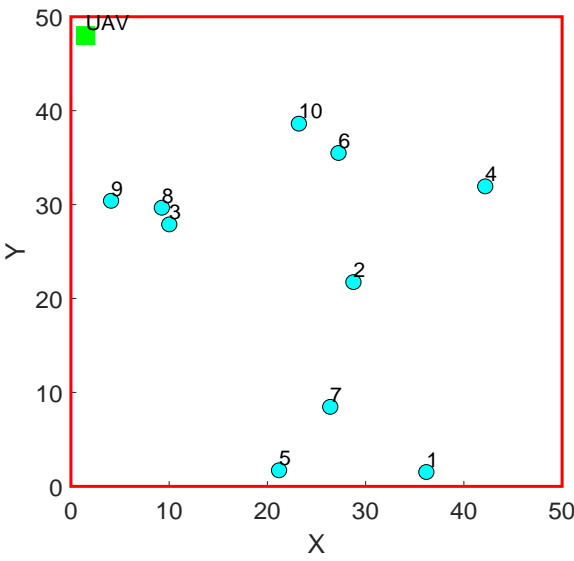

(a) System model.

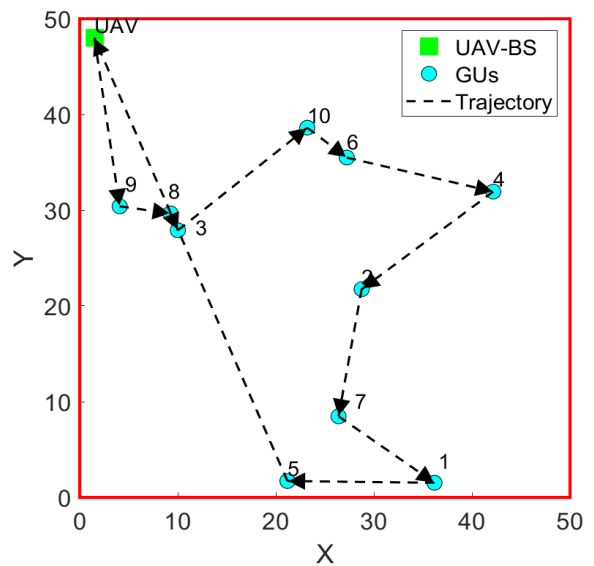

(c) Algorithm 1.

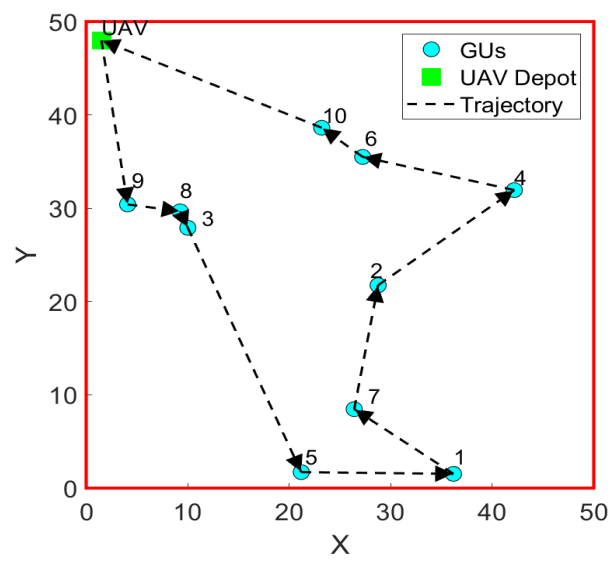

(b) Traditional Traveling Salesman Problem.

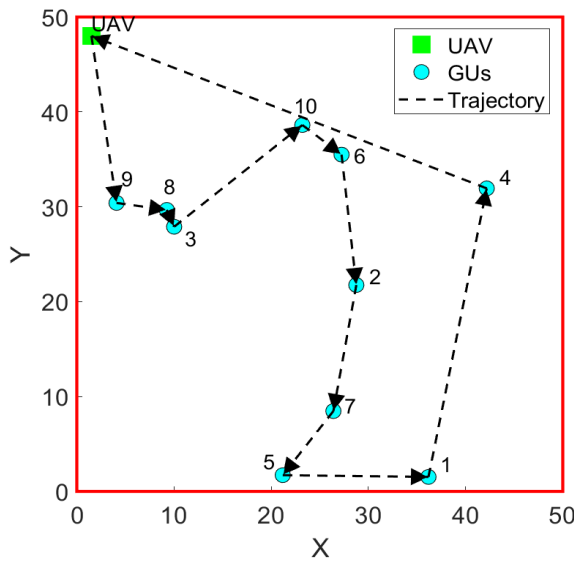

(d) Algorithm 2.

Fig. 1: Comparison of UAV's trajectories with different path designs.

hovering, and communications, respectively, where $P_{f l y}(v)$ is provided in (4) and $v_{h o v}$ is the minimum velocity of the UAV when hovering, e.g., $v_{\text {hov }}=5 \mathrm{~m} / \mathrm{s}$ [14].

\section{Minimization OF THE TOTAL TRAVELING TIME OF THE UAV}

In this section, we design the UAV trajectory that minimizes the total UAV's traveling time while satisfying the RT constraints for all GUs. Intuitively, we aim at finding the visiting order $\mathbf{u} \triangleq\left[u_{1}, \ldots, u_{K}\right]$ and the UAV velocities which result in the smallest traveling time. The problem is formulated as follows:

$$
\begin{array}{ll}
\mathcal{P}_{1}: & \min _{\mathbf{u},\left\{v_{k}\right\}_{k=1}^{K+1}} \sum_{k=1}^{K+1} \frac{l_{u_{k-1} \rightarrow u_{k}}}{v_{k}} \\
\text { s.t. } & \mathrm{C} 1: \sum_{i=1}^{k}\left(\frac{l_{u_{i-1} \rightarrow u_{i}}}{v_{i}}+\tau_{i}\right) \leq \eta_{u_{k}}, 1 \leq k \leq K \\
& \mathrm{C} 2: v_{k} \leq V_{\max }, \forall k,
\end{array}
$$

where $l_{u_{k-1} \rightarrow u_{k}}$ is defined in (1), $V_{\max }$ is the maximum velocity of the UAV and $u_{K+1} \equiv 0$ for convenience. In $\mathcal{P}_{1}$, constraint $\mathrm{C} 1$ guarantees the RT requirement for the GUs that states that the maximum latency to serve GU $u_{k}$ cannot exceed the predefined RT $\eta_{u_{k}}$.

\section{A. Algorithm 1: Exhaustive search algorithm}

In this subsection, we propose the exhaustive search algorithm to solve (7). The principle of the exhaustive search algorithm is to visit all the paths (trajectories) and find the shortest Hamiltonian cycle path [15] while satisfying the RT constraint.

Lemma 1: Problem $\mathcal{P}_{1}$ is equivalent to problem $\mathcal{P}_{2}$, where

$$
\begin{aligned}
& \mathcal{P}_{2}: \min _{\mathbf{u} \in \mathcal{I}} \sum_{k=1}^{K+1} \frac{l_{u_{k-1} \rightarrow u_{k}}}{V_{\max }} \\
& \text { s.t. } \mathrm{C} 1: \sum_{i=1}^{k}\left(\frac{l_{u_{i-1} \rightarrow u_{i}}}{V_{\max }}+\tau_{i}\right) \leq \eta_{u_{k}}, 1 \leq k \leq K,
\end{aligned}
$$

where $\mathcal{I}$ denotes all the permutations of $(1,2, \ldots, K)$.

The proof of Lemma 1 can be found by considering the monotonic of both the objective function and constraint $\mathrm{C} 1$ in $\mathcal{P}_{1}$ with respect to $v_{k}, \forall k$. Indeed, let $\mathbf{u}^{\star},\left\{v_{k}^{\star}\right\}_{k=1}^{K}$ denote the optimal solutions of problem $\mathcal{P}_{1}$ and suppose that is at least one velocity $v_{1}^{\star}$, without loss of generality, less than $V_{\max }$, 


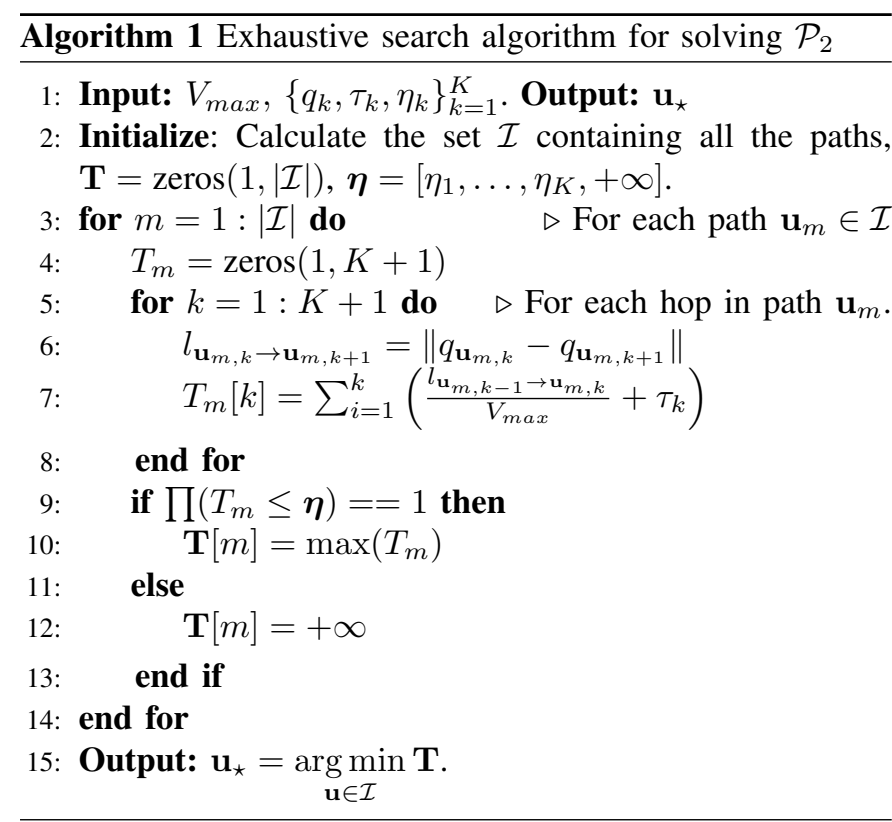

i.e., $v_{1}^{\star}<V_{\max }$. Now consider a candidate $\mathbf{u}^{\star}$ and $\left[v_{1}^{+}=\right.$ $\left.V_{\max }, v_{2}^{\star}, \ldots, v_{K}^{\star}\right]$, which satisfy both constraints $\mathrm{C} 1$ and $\mathrm{C} 2$. In addition, this candidate achieve a smaller objective function since $\frac{l_{u_{0} \rightarrow u_{1}}}{V_{\max }}<\frac{l_{u_{0} \rightarrow u_{1}}}{v_{1}^{\star}}$, which contradicts to the assumption of the optimality of $\left\{v_{k}^{\star}\right\}_{k=1}^{K}$. Thus, $\mathcal{P}_{1}$ achieves the optimal value at $v_{k}^{\star}=V_{\max }, \forall k$.

It is observed that problem $\mathcal{P}_{2}$ is in a form of TSPTW problem, which can be solved by finding the minimum cost tour starting and ending at location 0 and visiting all GUs only once. The steps to solve (7) is summarized in Algorithm 1. Basically, Algorithm 1 will try all valid paths which visit all the GUs once and calculate the visit time. It then compares the visit time to every GU with the corresponding RT requirements (constraint $\mathrm{C} 1$ in (7)). The best trajectory which satisfies all the RT constraints and imposes the minimum traveling time will be selected.

\section{B. Algorithm 2: Heuristic search algorithm}

Although providing the global optimality, the high computation complexity of Algorithm 1 may limit its potential in realistic scenarios. In this subsection, we propose a heuristic search algorithm, which compromises the performance and complexity. The key idea behind the heuristic algorithm is to refine the search space at each step, in which it only foresees one hop ahead when checking the RT condition. The searching in the heuristic algorithm consists of $K$ steps, in which it maintains two sets: a set of visited GUs and another set of GUs which have not been visited. At the $k$-th step, it only looks for the best GU to be added to the trajectory. This GU is then added into the visited set and excluded from the not-visited set. The complexity of the heuristic algorithm is $\mathcal{O}\left(K^{2}(K-1) / 2\right)$, which is significantly smaller than the complexity $\mathcal{O}(K \times K !)$ of Algorithm 1. Details of the heuristic algorithm are described in Algorithm 2.

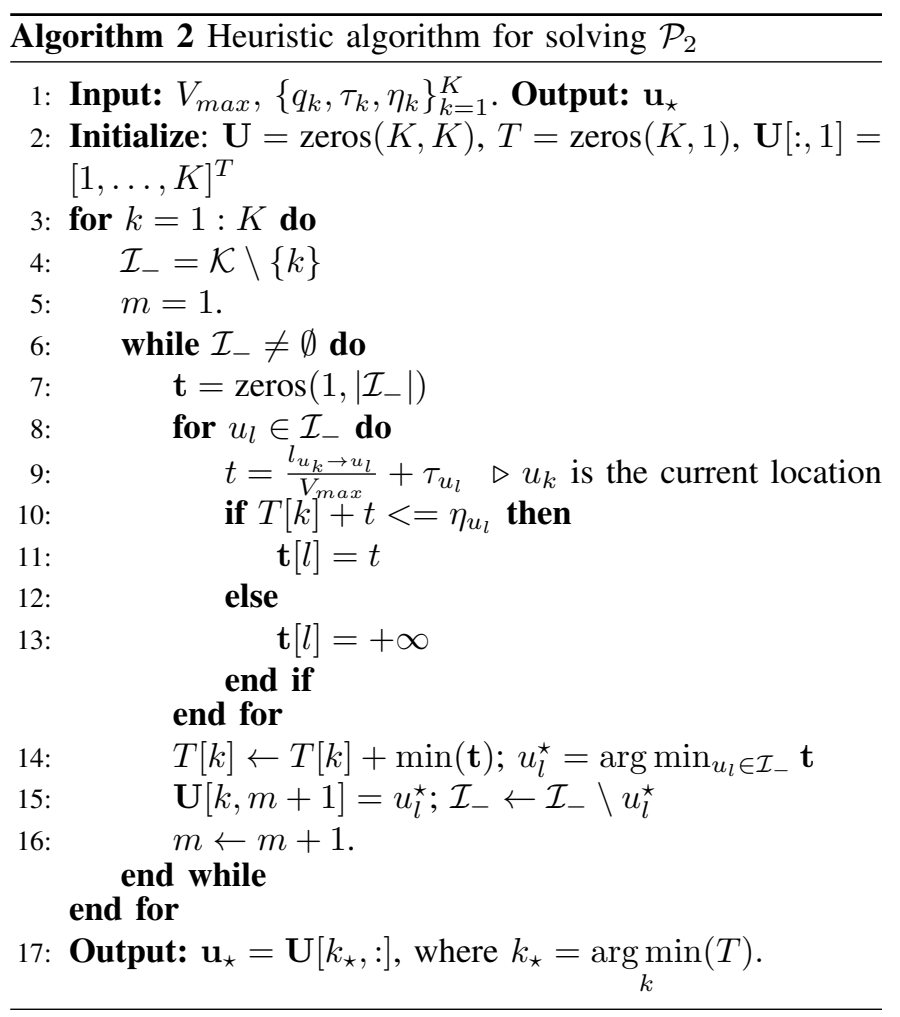

\section{Minimization OF THE UAV's EnERGY CONSUMPTION}

The previous section designs the trajectory based on the UAV maximum speed. While this method is preferred to minimize the traveling time, it might not energy-efficient since it over-estimate the RT constraints. In this section, we minimize total energy consumption of the UAV via the joint optimization of the UAV velocity over the given trajectory, e.g., the output of Algorithms 1 and 2. The energy minimization problem is formulated as

$$
\begin{aligned}
\mathcal{P}_{3}: \min _{\left\{v_{k}\right\}_{k=1}^{K+1}} & \sum_{k=1}^{K+1}\left(E_{f l y, k}+E_{\text {hov }, k}+E_{\text {com }, k}\right) \\
\text { s.t. } \quad & \mathrm{C} 1 \text { and } \mathrm{C} 2 \text { in }(6), \\
& \mathrm{C} 3:\left|v_{k}-v_{k-1}\right| \leq \Delta V, \forall k
\end{aligned}
$$

where the objective function is provided in Section II-B and constraint C3 set the limit on the velocity change between adjacent GUs.

Because $E_{c o m, k}$ and $E_{h o v, k}$ are independent from $v_{k}$, they can be removed from the objective function of (8) without loss of generality. By reformulated constraint $\mathrm{C} 3$ equivalently as

$$
v_{k}-v_{k-1} \leq \Delta V \text { and } v_{k-1}-v_{k} \leq \Delta V
$$

it is straightforward to prove the convexity of $\mathrm{C} 3$. In addition, since function $\frac{1}{x}$ is convex in $\mathbb{R}^{+}$, constraint $\mathrm{C} 1$ in (6) is convex. The most challenge lays in $E_{f l y, k}$ in the objective function.

Lemma 2: The energy consumption $E_{f l y, k}$ is convex. 
Proof: From (4) we have

$$
\begin{aligned}
E_{f l y, k}(v)=P_{0} l_{k}\left(\frac{1}{v}+\alpha_{1} v\right) & +P_{1} l_{k} \sqrt{\sqrt{v^{-4}+\alpha_{2}^{2}}-\alpha_{2}} \\
& +\alpha_{3} l_{k} v^{2}
\end{aligned}
$$

where $l_{k}$ is the distance in hop $k$ and other parameters are defined in Table I. The second derivative of $E_{f l y, k}(v)$, after some manipulations, can be expressed as follows

$$
\begin{aligned}
& \frac{d^{2}}{d v^{2}} E_{f l y, k}=\frac{2 P_{0} l_{k}}{v^{3}}+2 \alpha_{3} l_{k}+P_{1} l_{k} \beta, \text { where } \\
& \beta=\frac{1}{v^{6} \sqrt{\alpha_{2}^{2}+v^{-4}} \sqrt{\sqrt{\alpha_{2}^{2}+v^{-4}}-\alpha_{2}}} \times \\
& (5-\frac{2}{1+\alpha_{2}^{2} v^{4}}-\underbrace{\frac{1}{\alpha_{2}^{2} v^{4}+1-\alpha_{2} v^{2} \sqrt{\alpha_{2}^{2} v^{4}+1}}}_{\beta_{1}},
\end{aligned}
$$

Denote $X=\alpha_{2} v^{2} \geq 0$, then we can express $\beta_{1}=$ $\alpha_{2}^{2} v^{4}+1-\alpha_{2} v^{2} \sqrt{\alpha_{2}^{2} v^{4}+1}=X^{2}+1-X \sqrt{X^{2}+1}$. Since $X \sqrt{X^{2}+1} \leq \frac{2 X^{2}+1}{2}$, it yields

$$
\beta_{1} \geq X^{2}+1-\frac{2 X^{2}+1}{2}=\frac{1}{2} \text {. }
$$

In addition, since $1+\alpha_{2}^{2} v^{4} \geq 1$, we obtain the second term in (11) is always greater than or equal to 1 . Thus, $\beta>0, \forall v$. Since $P_{0}, l_{k}, \alpha_{3}$ are also positive, from (10) we conclude that the second derivative of $E_{f l y, k}(v)$ is alway positive, which proves the convexity of $E_{f l y, k}$.

By using Lemma 2, we observe that problem $\mathcal{P}_{3}$ is convex since the objective and all constraints are convex. Therefore, it can be efficiently solved by a standard method, e.g., gradient descent.

\section{Numerical Results}

This section provides numerical results to validate the proposed designs. Unless otherwise stated, the parameters are set as follows: $H=50 \mathrm{~m}, B=5 \mathrm{MHz}$, path loss exponent $\alpha=2, \sigma^{2}=-90 \mathrm{dBm}, P_{\text {com }}=0.1 \mathrm{~mW}, \tau_{k}=0.131 \mathrm{~s}$, UAV's coverage area is $50 \mathrm{~m} \times 50 \mathrm{~m}$, UAV ground station is located at $(1.5 \mathrm{~m}, 48 \mathrm{~m})$. On a more general level, we perform 1000 independent trials of Monte-Carlo simulations. In details, for each iteration, we deploy a different distribution of GUs' topology and RT constraints. The proposed solutions are compared with a solution in [8], [11], which is based on the TSP.

We first evaluate the proposed trajectory designs via the outage probability metric (OP), which is defined as the probability that no feasible path (a path that satisfies all the GUs' RT requirements) is found. Fig. 2 presents the OP of the proposed algorithms and the reference as a function of $V_{\max }$ with the RT requirements $\eta_{k}$ ranging between 3 and 10 seconds. It is shown that the proposed algorithms significantly improve the OP compared with the reference for all values of $V_{\max }$. Specifically, at $V_{\max }=40 \mathrm{~m} / \mathrm{s}$, the exhaustive search algorithm always find the trajectory that satisfies all the GUs'

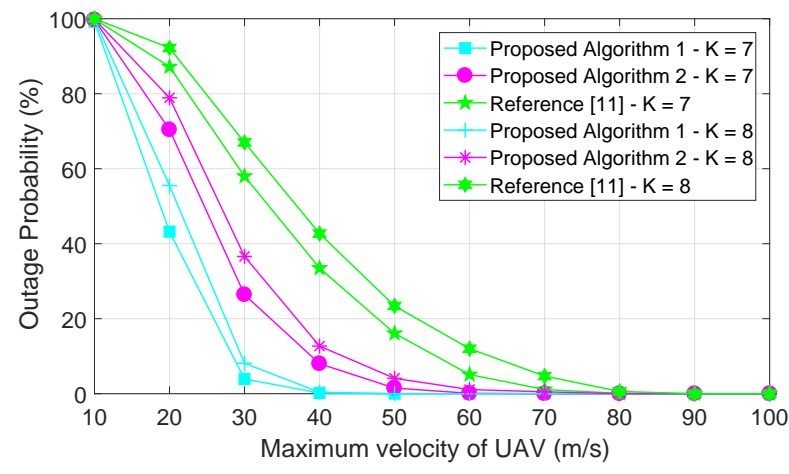

Fig. 2: Average OP (\%) versus $V_{\max }(\mathrm{m} / \mathrm{s})$.

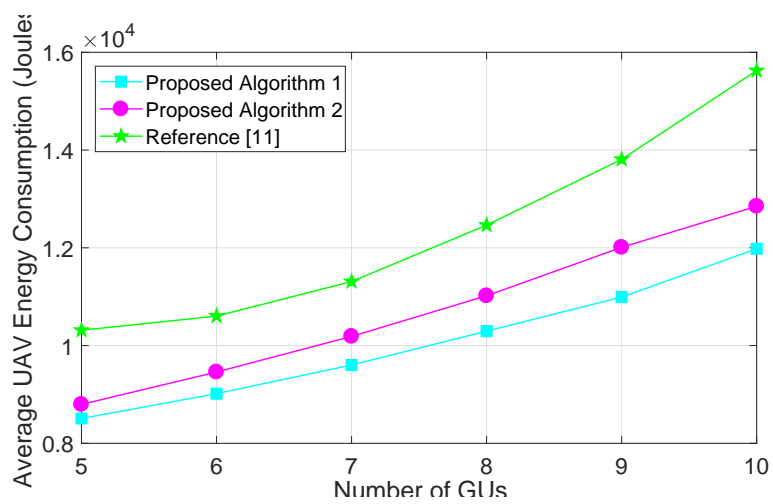

Fig. 3: Average energy consumption vs. number of GUs.

RT constraints and the heuristic-based algorithm achieves less than $10 \%$ OP. Whereas the reference scheme imposes $38 \%$ OP. The OP of all schemes can be reduced by by increasing $V_{\max }$, which is because a higher $V_{\max }$ results in lower traveling time between the GUs. Consequently, it is highly probable for the UAV to satisfy the GUs' RT. When $V_{\max }$ is sufficiently large, all the schemes achieve zero OP.

Next, we examine the energy consumption of the proposed optimization in Section IV and compare with the TSP-based reference scheme in [8], [11]. For a fair comparison, we assume that $V_{\max }$ is sufficiently large so that all schemes have at least one feasible trajectory. Once the best trajectory is obtained based on the proposed Algorithms 1 and 2, we apply the optimization $\mathcal{P}_{3}$ to minimize the total UAV's energy consumption. Fig. 3 plots the energy consumption (joules) of all schemes as a function $K$ - the number of GUs. A similar observation is that our proposed designs significantly reduce the UAV's consumed energy compared with the reference. This is due to the fact that the reference (TSP-based) TSP always selects the shortest path regardless of the GUs' RT requirements. Consequently, in order to satisfy all GUs' RT constraint, the UAV (in this case) has to fly with higher velocity than in our proposed designs. Obviously, serving more GUs requires more energy consumption, as shown in the figure.

Fig. 4 compares the running time (seconds) as a function of the number of GUs. Clearly, the exhaustive search (Algorithm 1) imposes the largest running time, which increases expo- 


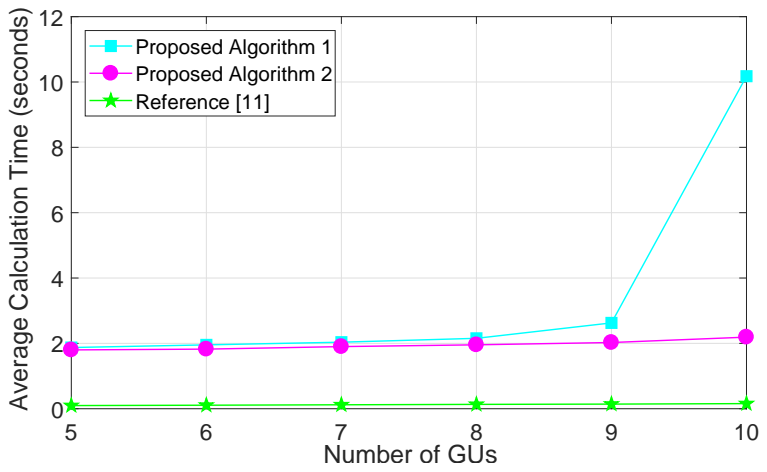

Fig. 4: Average calculation time versus number of GUs.

nentially with the network size, as it tries all possible paths. The heuristic search (Algorithm 2) consumes much less time compared with Algorithm 1. More importantly, its running time is at a constant gap from the TSP-based reference. From practical aspects, Algorithm 2 is preferred as it has a relatively small complexity while achieving good performance. Although having the smallest running time, the TSP-based reference has a poor performance, which is far worse than the proposed Algorithms, as shown in Fig. 2 and 3.

\section{CONCLUSION}

We have investigated the energy-efficient trajectory design for UAV-assisted communications networks which take into consideration latency requirements from the GUs. We proposed two algorithms for UAV trajectory design based on the TSPTW in order to minimize the UAV's traveling time while satisfying the GUs' latency constraints. Based on the obtained trajectory, we minimized the total energy consumption via a joint design of the UAV's velocity in all hops. It was shown via numerical results that our proposed designs outperform the reference scheme in terms of both energy consumption and outage probability.

The outcome of this work motivates future works in UAV communications networks. One problem is to jointly select the paths and optimize the velocity, which requires advanced optimization techniques but might further improve the UAV's performance. Another promising problem is to consider dynamic network topology. In this case, an adaptive solution that optimizes the UAV trajectory on the fly is required.

\section{ACKNOWLEDGEMENT}

This research is supported by the Luxembourg National Research Fund under project FNR CORE ProCAST, grant C17/IS/11691338.

\section{REFERENCES}

[1] D. Lopez-Perez, I. Guvenc, G. de la Roche, M. Kountouris, T. Q. S. Quek, and J. Zhang, "Enhanced intercell interference coordination challenges in heterogeneous networks," IEEE Wireless Communications, vol. 18 , no. 3, pp. 22-30, June 2011.

[2] T. X. Vu, H. D. Nguyen, T. Q. S. Quek and S. Sun, "Adaptive Cloud Radio Access Networks: Compression and Optimization," IEEE Transactions on Signal Processing, vol. 65, no. 1, pp. 228-241, 1 Jan.1, 2017.

[3] T. X. Vu, T. V. Nguyen, and T. Q.S. Quek, "Power Optimization with BLER Constraint for Wireless Fronthauls in C-RAN," IEEE Communication Letter, vol. 20, no. 3, pp. 602-605, Mar. 2016.

[4] M. Mozaffari, W. Saad, M. Bennis, M. Debbah, "Wireless communication using unmanned aerial vehicles (UAVs): Optimal transport theory for hover time optimization," IEEE Transactions on Wireless Communications, vol. 16, no. 12, pp. 8052-8066, Dec. 2017.

[5] M. Erdelj, E. Natalizio, K. R. Chowdhury, I. F. Akyildiz, "Help from the sky: Leveraging UAVs for disaster management", IEEE Pervasive Computing, vol. 16, no. 1, pp. 24-32, Mar. 2017.

[6] K. Li, R.C. Voicu, S.S. Kanhere, W. Ni, E. Tovard, "Energy Efficient Legitimate Wireless Surveillance of UAV Communications," IEEE Transactions on Vehicular Technology, vol. 68 , no. 3, pp. 2283 - 2293, Mar. 2019.

[7] M. Bacco, A. Berton, A. Gotta, L. Caviglione, "IEEE 802.15.4 AirGround UAV Communications in Smart Farming Scenarios," IEEE Communications Letters, vol. 22, no. 9, pp. 1-4, Sept. 2018.

[8] Y. Zeng, X. Xu, and R. Zhang, "Trajectory design for completion time minimization in UAV-enabled multicasting," IEEE Transactions on Wireless Communications, vol. 17, no. 4, pp. 2233-2246, Apr. 2018.

[9] Z. Cheng, Y. Zeng, and R. Zhang, "Energy-efficient data collection in UAV enabled wireless sensor network," IEEE Communication Letter, vol. 7, no. 3, pp. 328-331, Jun. 2018.

[10] D. Yang, Q. Wu, Y. Zeng, and R. Zhang, "Energy trade-off in groundto-UAV communication via trajectory design," IEEE Transactions on Vehicular Technology, vol. 67, no. 7, pp. 6721-6726, Jul. 2018.

[11] Y. Zeng, J. $\mathrm{Xu}$, and R. Zhang, "Energy minimization for wireless communication with rotary-wing UAV," submitted to IEEE Transactions on Wireless Communications, available online at https://arxiv.org/abs/1804.02238.

[12] T. X. Vu, S. Chatzinotas, B. Ottersten and T. Q. Duong, "Energy Minimization for Cache-Assisted Content Delivery Networks With Wireless Backhaul," IEEE Wireless Communications Letters, vol. 7, no. 3, pp. 332-335, June 2018.

[13] D. W. Matolak and R. Sun, "Unmanned aircraft systems: air-ground channel characterization for future applications," IEEE Vehicular Technology Magazine, vol. 10, no. 2, pp. 79-85, Jun. 2015.

[14] Q. Wu, L. Liu, R. Zhang, "Fundamental tradeoffs in communication and trajectory design for UAV-enabled wireless network," IEEE Wireless Communications, vol. 26, no. 1, pp. 36-44, 2019.

[15] B. Bollobas, "Graph theory: an introductory course," Springer Science \& Business Media, vol. 63, 2012.

[16] S. Boyd and L. Vandenberghe, Convex Optimization. Cambridge, U.K.: Cambridge Univ. Press, 2004. 